\title{
Antibiotika bei akuter Bronchitis ohne Vorteil
}

\section{Bei einem akuten unkomplizierten Infekt der tiefen Atemwege lässt sich kein Vorteil für eine antibiotische Be- handlung finden.}

- In einer großen randomisierten Studie, die den Effekt einer antibiotischen Behandlung bei akuten unkomplizierten lnfekten der tiefen Atemwege untersuchen sollte, wurde älteren Patienten besondere Beachtung geschenkt, da diese vermehrt an Begleiterkrankungen leiden und somit möglicherweise eher von einer antibiotischen Behandlung profitieren könnten. Insgesamt wurden 3108 Patienten eingeschlossen. Davon erhielten 2061 Patienten randomisiert Amoxicillin oder Placebo. Ein knappes Drittel der eingeschlossenen Patienten war älter als 60 Jahre.
Die mediane Zeit bis zur vollständigen subjektiven Ausheilung betrug sechs Tage in der Amoxicllingruppe und sieben Tage in der Placebogruppe, wobei dieser Unterschied in der statistischen Analyse nicht signifikant war $(\mathrm{p}=0,229)$. Auch hinsichtlich der Schwere der Symptome im Verlauf und dem Auftreten von klinischen Verschlechterungen fanden sich keine entscheidenden Unterschiede zwischen den Gruppen. Gleiches fand sich auch bei der gesonderten Analyse der Patienten $>60$ Jahre.

\footnotetext{
- P. Little et al.

Amoxicillin for acute lower-tract infection in primary care when pneumonia is not suspected: a 12-country, randomized, placebocontrolled trial. Lancet Infect Dis. 2013 Feb; 13(2):123-129
}

\section{Kommentar}

Diese Studie zeigt einmal mehr, dass sich bei einem akuten unkomplizierten Infekt der tiefen Atemwege kein Vorteil für eine antibiotische Behandlung finden lässt. Einer der Hauptgründe ist sicherlich, dass der Großteil dieser Erkrankungen durch Viren verursacht wird. Deshalb sollte im Alltag bei diesen Patienten auf eine Antibiotikaverordnung verzichtet werden, insbesondere um einer unnötigen Resistenzentwicklung vorzubeugen. Wichtig ist allerdings, dass Patienten mit einer Pneumonie nicht übersehen werden, da diese meist bakteriell verursacht ist und in der Regel eine antibiotische Behandlung erfordert.

S. R. OTT =

\section{Pseudohernie durch Zoster}

— Bei einem 75-jährigen Mann entwickelte sich ein bläschenartiger Ausschlag im Bereich der Dermatome Th11 und Th12 rechts. Die Diagnose eines Herpes zoster war leicht gestellt und der Patient wurde entsprechend behandelt.

Vier Wochen später war der Ausschlag zwar wesentlich gebessert (Abb. A), der Patient bemerkte aber eine Vorwölbung seiner Bauchwand auf der rechten Seite verbunden mit einer
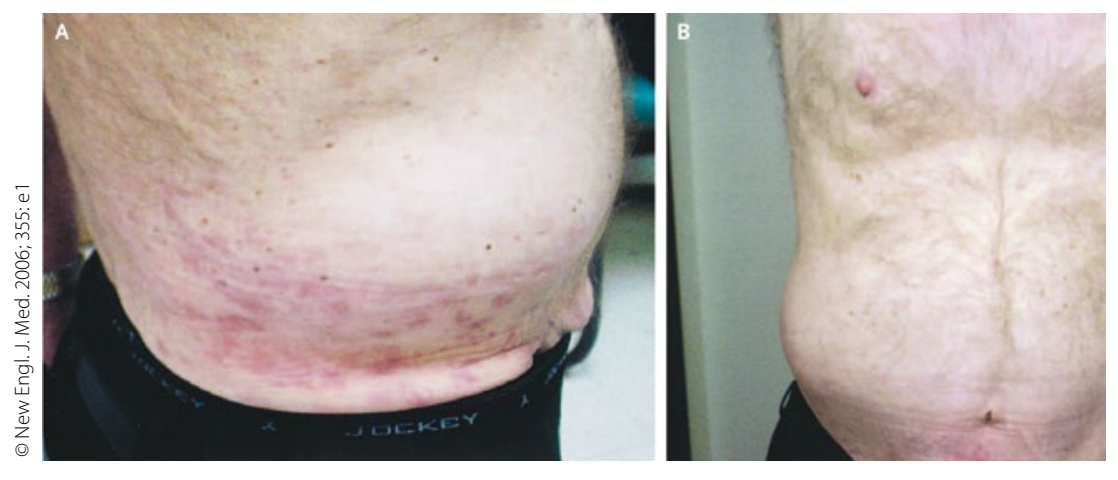
eine Pseudohernie der Bauchwand im Ausmaß von $5 \times 5 \mathrm{~cm}$ dar. Der Patient wies eine ausgeprägte Hyperästhesie der darüber liegenden Haut auf. Bei der Rumpfbeugung bemerkte man eine Bewegung des Nabels nach oben und links. Ein MRT des Abdomens zeigte keine echte Hernie oder Raumforderung. Elektromyografisch stellte man

Vorwölbung der Bauchwand nach Zoster-Manifestation.

denervierende Veränderungen des $\mathrm{M}$. paraspinalis und $M$. infraumbilicalis rechts fest. Ein MRT der Brust- und Lendenwirbelsäule war unauffällig.

\section{Kommentar}

Der Herpes Zoster ist eine Viruserkrankung der Ganglien der Hinterwurzeln und sensorischer Nervenfasern, der durch ein einseitiges bläschenartiges Exanthem der Haut und Schmerzempfindungen im Bereich des entsprechenden Dermatoms gekennzeichnet ist. Eine segmentale motorische Schwäche ist eine ungewöhnliche Komplikation der Erkrankung, die nur in 3-5\% der Fälle von Herpes zoster auftritt. Die Prognose auch für diese Komplikation ist gut.

H. S. FÜESSL - 